\title{
Leukemia inhibitory factor protects the lung during respiratory syncytial viral infection
}

\author{
Robert F Foronjy, Abdoulaye J Dabo, Neville Cummins and Patrick Geraghty*
}

\begin{abstract}
Background: Respiratory syncytial virus (RSV) infects the lung epithelium where it stimulates the production of numerous host cytokines that are associated with disease burden and acute lung injury. Characterizing the host cytokine response to RSV infection, the regulation of host cytokines and the impact of neutralizing an RSV-inducible cytokine during infection were undertaken in this study.

Methods: A549, primary human small airway epithelial (SAE) cells and wild-type, TIR-domain-containing adapter-inducing interferon- $\beta$ (Trif) and mitochondrial antiviral-signaling protein (Mavs) knockout (KO) mice were infected with RSV and cytokine responses were investigated by ELISA, multiplex analysis and qPCR. Neutralizing anti-leukemia inhibitory factor (LIF) IgG or control lgG was administered to a group of wild-type animals prior to RSV infection.
\end{abstract}

Results and discussion: RSV-infected A549 and SAE cells release a network of cytokines, including newly identified RSV-inducible cytokines LIF, migration inhibitory factor (MIF), stem cell factor (SCF), CCL27, CXCL12 and stem cell growth factor beta (SCGF- $\beta$ ). These RSV-inducible cytokines were also observed in the airways of mice during an infection. To identify the regulation of RSV inducible cytokines, Mavs and Trif deficient animals were infected with RSV. In vivo induction of airway IL-1 $\beta, I L-4, I L-5, I L-6, I L-12(p 40), I F N-\gamma, C C L 2, C C L 5, C C L 3, C X C L 1$, IP-10/CXCL10, IL-22, MIG/CXCL9 and MIF were dependent on Mavs expression in mice. Loss of Trif expression in mice altered the RSV induction of IL-1 $\beta, I L-5, C X C L 12$, MIF, LIF, CXCL12 and IFN- . Silencing of retinoic acid-inducible gene-1 (RIG-I) expression in A549 cells had a greater impact on RSV-inducible cytokines than melanoma differentiation-associated protein 5 (MDA5) and laboratory of genetics and physiology 2 (LGP2), and Trif expression. To evaluate the role of LIF in the airways during RSV infection, animals were treated with neutralizing anti-LIF IgG, which enhanced RSV pathology observed with increased airspace protein content, apoptosis and airway hyperresponsiveness compared to control IgG treatment.

Conclusions: RSV infection in the epithelium induces a network of immune factors to counter infection, primarily in a RIG-I dependent manner. Expression of LIF protects the lung from lung injury and enhanced pathology during RSV infection.

Keywords: Respiratory syncytial virus, Immune response, Pathogen recognition receptors, Gene expression

\section{Background}

Respiratory syncytial virus (RSV) is a major respiratory pathogen, which typically infects the airway epithelium [1]. Those infected with RSV, usually infants, the elderly and immunocompromised patients but also healthy adults [2,3], develop mild to severe cough and dyspnea [4]. Wheezing and asthma symptoms are observed following

\footnotetext{
*Correspondence: pgeraghty@chpnet.org

Mount Sinai Roosevelt Hospital, Mt. Sinai Health System, Division of Pulmonary and Critical Care Medicine, New York, NY, USA
}

severe RSV lower respiratory tract infection [5]. RSV infection occur frequently in the respiratory tract of individuals with an underlying lung disease, such chronic obstructive pulmonary disease (COPD) [6]. A range of inflammatory responses are associated with RSV infection, such as the induction of proinflammatory cytokines, chemokines and growth factors, such as GRO $\alpha$ / CXCL1, IL-1 $\beta$, IL1-RA, IL-2, IL-6, IL-7, CXCL8/IL-8, IL-9, CXCL10/IP-10, CXCL11, IL-15, IL-18, CCL2/MCP-1, CCL3/MIP-1 $\alpha$, CCL4/MIP-1 $\beta$, IFN- $\gamma$, CCL5/RANTES, 
epidermal growth factor (EGF), hepatocyte growth factor (HGF), granulocyte colony-stimulating factor (G-CSF), GM-CSF, fibroblast growth factor (FGF), vascular endothelial cell growth factor (VEGF) and tumor necrosis factor- $\alpha$ (TNF- $\alpha$ ) [1,7-16]. The importance of this cytokine response is underscored by the fact that increased levels of IL-1 $\beta$, IL1-RA, IL-6, IL-7, IL-8, G-CSF, EGF and HGF are associated with RSV infection severity $[10,15]$. Others have found that SNPs in IL-19, IL-20, MUC5AC, TNFRSF1B, C3, CTLA4, CXCL9, IL4R, and IL-7 genes are associated with recurrent wheezing following RSV infections [17]. Although RSV-induced cytokines have been studied extensively, the signaling mechanisms that regulate their expression and whether specific host cytokines contribute to airway injury or inflammation resolution remain incompletely understood.

Pathological studies demonstrate that RSV primarily infects airway epithelial cells [18]; therefore it is conceivable that a significant component of immune signaling associated with RSV infections originates from airway epithelial responses. Cytokines, chemokines and growth factors are expressed in these cells in response to microbial stimuli $[19,20]$ with numerous pathogen recognition receptors playing a major role in cytokine responses. Pathogen recognition receptors, such as TLRs and retinoic acid-inducible gene-1 (RIG-I)-like receptors [21], induce major signaling cascades following viral stimulation [22]. Indeed, the viral load of RSV correlates with RIG-I mRNA levels [23]. TLR3, $-7,-8$ and -9 recognize nucleic acid ligands but TLR4 proteins also bind to a major viral antigen of RSV F (fusion) protein [24]. Laboratory of genetics and physiology 2 (LGP2) and melanoma differentiation-associated protein-5 (MDA5) equally could be associated with RSV-induced immune responses [25]. Additionally, ligands for nucleotide-binding oligomerization domain-containing protein (NOD)-like receptors (NLR), such as NOD2, enhance TLR-ligandinduced activation and triggering such signaling cascades are plausible means of developing RSV-specific immunity [26]. Therefore, RSV can induce several pathogen recognition receptors resulting in multiple host immune responses.

In this study we employed in vitro and in vivo approaches to evaluate the cytokine/chemokine response to RSV infection. Normal human small airway epithelial (SAE) cells and A549 cells were utilized to examine 42 cytokine responses following RSV stimuli. Several newly identified RSV-inducible cytokines (leukemia inhibitory factor (LIF), migration inhibitory factor (MIF), stem cell factor (SCF), CCL27, CXCL12 and stem cell growth factor beta (SCGF- $\beta$ )) were induced in airways cells in vitro and in mouse lungs during a viral infection in vivo. The influence of TIR-domain-containing adapter-inducing interferon- $\beta$ (Trif) and mitochondrial antiviral-signaling protein (MAVS) signaling pathways were examined on RSV-induced cytokine levels in mice and A549 cells. The significance of LIF, an IL-6 cytokine family member, expression during RSV was also determined. LIF is primarily recognized for its ability to preserve the totipotency of embryonic stem cells [27] and is detected in acute respiratory distress syndrome (ARDS) [28] but recently LIF was described to protect the lung from injury during pneumonia [29]. Utilizing neutralizing anti-LIF IgG, LIF was observed to play a critical role in protecting the lung from injury during RSV infection. Our findings indicate that RSV infection induces a significant host cytokine response associated with viral clearance and airway protection.

\section{Results \\ SAE and A549 cells secrete similar cytokines following RSV stimuli}

A human cytokine array was used to simultaneously detect the levels of 36 different cytokines, chemokines, and acute phase proteins in A549 cells treated with mock and RSV for 24 hours. Protein array analysis identified that a large number of cytokines are released from A549 cells upon RSV stimuli (Additional file 1: Figure S1). The most striking RSV inducible cytokines were IL-6, IL-8, MCP-1/CCL2, MIF, GRO $\alpha$ CXCL1, and RANTES/CCL5 (Additional file 1: Figure S1). To complement the cytokine array analysis, multiplex analysis was performed to examine 42 cytokines, chemokines and growth factors released from A549 cells, in addition to human SAE cells. Both SAE and A549 cells released similar cytokines in response to RSV infection (Table 1). In both A549 and SAE cells, RSV significantly induced IL-1 $\alpha$, IL- $1 \beta$, IL-1RA, IL-6, IL-7, IL-8, CXCL1, CCL11, fibroblast growth factor (FGF), G-CSF, IFN- $\gamma$, CXCL10, CCL2, MIF, platelet-derived growth factor (PDGF-BB), TNF- $\alpha$, VEGF, LIF, CCL27, CXCL12, SCGF- $\beta$ and SCF release into media. Interestingly, SAE cells also released IL-16, IL-18, CXCL9, CCL7 and HGF upon RSV stimulation. A549 cells differed to SAE cells in releasing IL-10, IL-12(p70), GM-CSF, CCL3, CCL4 and TNF-related apoptosis-inducing ligand (TRAIL) upon RSV stimulation. RSV-inducible cytokines were examined following stimulation with UV-inactivated RSV in A549 cells, to determine if cytokines are induced following viral detection or viral replication. RANTES/CCL5, CXCL12, SCGF- $\beta$, CCL27, LIF, MIF, CXCL10, CXCL1, CCL2, IL-6, SCF, IL-7, TNF- $\alpha$ and G-CSF were all secreted following stimuli with live RSV only (Figure 1). IL-8 and IFN- $\gamma$ was secreted at significantly higher levels from UV-RSV treated cells compared to mock treated cells but at lower levels to live RSV (Figure 1A). Therefore, RSV replication induces a plethora of immune responses in airway epithelial cells. 
Table 1 RSV induces cytokine release from human airway epithelial cells

\begin{tabular}{|c|c|c|c|c|}
\hline \multirow[b]{3}{*}{ Cytokines (pg/ml media) } & \multicolumn{4}{|c|}{ Airway epithelial cell type } \\
\hline & \multicolumn{2}{|c|}{ SAE cells (Type I) } & \multicolumn{2}{|c|}{ A549 (Type II) } \\
\hline & Mock & RSV & Mock & RSV \\
\hline IL-1a & $12.66 \pm 1.76$ & $31.75 \pm 1.48^{*}$ & $13.52 \pm 0.80$ & $34.33 \pm 4.3^{*}$ \\
\hline IL-1 $\beta$ & $9.55 \pm 1.17$ & $15.50 \pm 2.45^{*}$ & $1.28 \pm 0.05$ & $2.28 \pm 0.45^{*}$ \\
\hline IL1-RA & $40.37 \pm 6.37$ & $63.25 \pm 4.82^{*}$ & $5.20 \pm 0.37$ & $23.28 \pm 3.88^{*}$ \\
\hline IL-2 & $0.98 \pm 0.28$ & $1.44 \pm 0.42$ & $0.20 \pm 0.05$ & $0.25 \pm 0.07$ \\
\hline IL-2RA & $41.65 \pm 3.57$ & $44.67 \pm 2.12$ & $36.72 \pm 0.58$ & $34.53 \pm 0.55$ \\
\hline IL-3 & $102.7 \pm 8.85$ & $100.2 \pm 7.37$ & $59.09 \pm 3.54$ & $58.08 \pm 4.56$ \\
\hline IL-6 & $2349 \pm 234.6$ & $3260 \pm 153.8^{*}$ & $17.21 \pm 0.79$ & $534.4 \pm 19.22^{*}$ \\
\hline IL-7 & $29.36 \pm 4.87$ & $55.37 \pm 8.32 *$ & $17.00 \pm 4.57$ & $34.44 \pm 4.15^{*}$ \\
\hline IL-8/CXCL8 & $2479 \pm 154.4$ & $5094 \pm 363.5^{*}$ & $142.2 \pm 20.70$ & $1203 \pm 83.75^{*}$ \\
\hline IL-10 & $6.89 \pm 1.31$ & $8.13 \pm 0.15$ & $5.85 \pm 0.27$ & $7.02 \pm 0.24^{*}$ \\
\hline IL-12(p40) & $193.4 \pm 17.18$ & $183.9 \pm 19.78$ & $127.2 \pm 10.11$ & $135.0 \pm 19.54$ \\
\hline IL-12(p70) & $36.76 \pm 5.48$ & $36.09 \pm 5.94$ & $14.54 \pm 1.29$ & $19.86 \pm 0.81^{*}$ \\
\hline IL-16 & $115.7 \pm 14.5$ & $223.5 \pm 19.04^{*}$ & $652.1 \pm 32.79$ & $387.5 \pm 45.97$ \\
\hline IL-18 & $6.70 \pm 0.71$ & $11.94 \pm 0.92^{*}$ & $6.24 \pm 0.31$ & $7.06 \pm 0.37$ \\
\hline GROa/CXCL1 & $8482 \pm 428$ & $11119 \pm 639^{*}$ & $2431 \pm 177.7$ & $9473 \pm 366.4^{*}$ \\
\hline Eotaxin/CCL11 & $5.16 \pm 0.24$ & $10.37 \pm 0.62^{*}$ & $1.38 \pm 0.16$ & $3.43 \pm 0.37^{*}$ \\
\hline FGF & $6.60 \pm 0.30$ & $10.72 \pm 1.41^{*}$ & $4.24 \pm 0.51$ & $13.92 \pm 2.16^{*}$ \\
\hline G-CSF/CSF-3 & $945.6 \pm 72.01$ & $1403 \pm 130.2$ & $1.00 \pm 0.79$ & $10.16 \pm 1.57^{*}$ \\
\hline GM-CSF/CSF-2 & $9.74 \pm 2.99$ & $10.04 \pm 3.15$ & $3.73 \pm 0.44$ & $7.12 \pm 0.51^{*}$ \\
\hline M-CSF/CSF-1 & $73.03 \pm 8.60$ & $98.50 \pm 6.41$ & $2.90 \pm 0.74$ & $4.55 \pm 0.63$ \\
\hline IFN-a2 & $4.62 \pm 0.32$ & $4.97 \pm 0.27$ & $4.46 \pm 0.04$ & $4.55 \pm 0.76$ \\
\hline IFN- $\gamma$ & $95.39 \pm 13.08$ & $171.6 \pm 12.04^{*}$ & $12.38 \pm 1.98$ & $81.81 \pm 8.88^{*}$ \\
\hline MIG/CXCL9 & $29.01 \pm 3.91$ & $61.41 \pm 2.99 *$ & $53.83 \pm 5.43$ & $104.81 \pm 12.11$ \\
\hline IP10/CXCL10 & $337.6 \pm 93.99$ & $10580 \pm 546^{*}$ & $5.13 \pm 0.35$ & $1836 \pm 97.5^{*}$ \\
\hline MCP-1/CCL2 & $146.2 \pm 15.51$ & $266.0 \pm 42.71 *$ & $557.1 \pm 30.48$ & $1088 \pm 56.54^{*}$ \\
\hline MCP-3/CCL7 & $71.68 \pm 8.97$ & $215.8 \pm 17.44^{*}$ & $96.03 \pm 10.57$ & $78.41 \pm 11.14$ \\
\hline MIP-1a/CCL3 & $1.88 \pm 0.12$ & $2.38 \pm 0.36$ & $1.25 \pm 0.09$ & $16.96 \pm 0.42^{*}$ \\
\hline MIP-1 $\beta / C C L 4$ & $1.55 \pm 0.32$ & $2.75 \pm 0.45$ & $0.77 \pm 0.13$ & $89.04 \pm 7.52^{*}$ \\
\hline MIF & $2123 \pm 186.2$ & $7435 \pm 179.5^{*}$ & $454.0 \pm 20.8$ & $1015 \pm 47.5^{*}$ \\
\hline PDGF-BB & $6.91 \pm 1.01$ & $16.90 \pm 2.98^{*}$ & $3.68 \pm 0.59$ & $10.23 \pm 0.16^{*}$ \\
\hline RANTES/CCL5 & $206.8 \pm 10.32$ & $339.9 \pm 29.54^{*}$ & $44.30 \pm 8.53$ & $656.9 \pm 82.5^{*}$ \\
\hline TNF-a & $16.78 \pm 0.57$ & $32.40 \pm 3.27^{*}$ & $1.14 \pm 0.12$ & $27.83 \pm 2.37^{*}$ \\
\hline TNF- $\beta /$ LTA & $21.98 \pm 1.39$ & $21.09 \pm 0.78$ & $17.59 \pm 0.18$ & $17.41 \pm 0.72$ \\
\hline VEGF & $3717 \pm 372.2$ & $5256 \pm 304.3^{*}$ & $1585 \pm 108.9$ & $3297 \pm 146.2^{*}$ \\
\hline TRAIL & $135.7 \pm 13.64$ & $209.2 \pm 6.82$ & $151.4 \pm 10.16$ & $191.9 \pm 5.06^{*}$ \\
\hline$\beta$-NGF & $23.92 \pm 1.76$ & $23.81 \pm 2.23$ & $20.50 \pm 1.17$ & $24.12 \pm 2.39$ \\
\hline LIF & $114.9 \pm 11.92$ & $227.4 \pm 6.7^{*}$ & $64.70 \pm 2.41$ & $212.7 \pm 15.88^{*}$ \\
\hline HGF & $63.33 \pm 4.98$ & $81.84 \pm 1.41^{*}$ & $71.23 \pm 1.47$ & $69.34 \pm 1.71$ \\
\hline CTAK/ CCL27 & $130.5 \pm 9.87$ & $249.3 \pm 9.73^{*}$ & $122.5 \pm 2.40$ & $211.7 \pm 8.91^{*}$ \\
\hline SDF-1a/CXCL12 & $672.0 \pm 31.7$ & $1504 \pm 97.33^{*}$ & $121.6 \pm 9.61$ & $359.4 \pm 28.6^{*}$ \\
\hline SCGF- $\beta$ & $685.7 \pm 32.1$ & $1000 \pm 73.1^{*}$ & $3575 \pm 191.2$ & $5905 \pm 161.9 *$ \\
\hline SCF & $19.09 \pm 1.08$ & $42.87 \pm 0.18^{*}$ & $27.50 \pm 1.52$ & $38.66 \pm 3.03^{*}$ \\
\hline
\end{tabular}

Cytokine levels were determined in cell culture supernatants from A549 or SAE cells 24 hours post RSV challenge, by multiplex analysis. Values are represented as mean media concentration $(\mathrm{pg} / \mathrm{ml}) \pm$ S.E.M. Assays were performed in triplicate from samples collected on separate days, where $n=10$ samples/group. Bold numbers denoted by *represents a p value less than 0.05 compared to mock treated cells. 


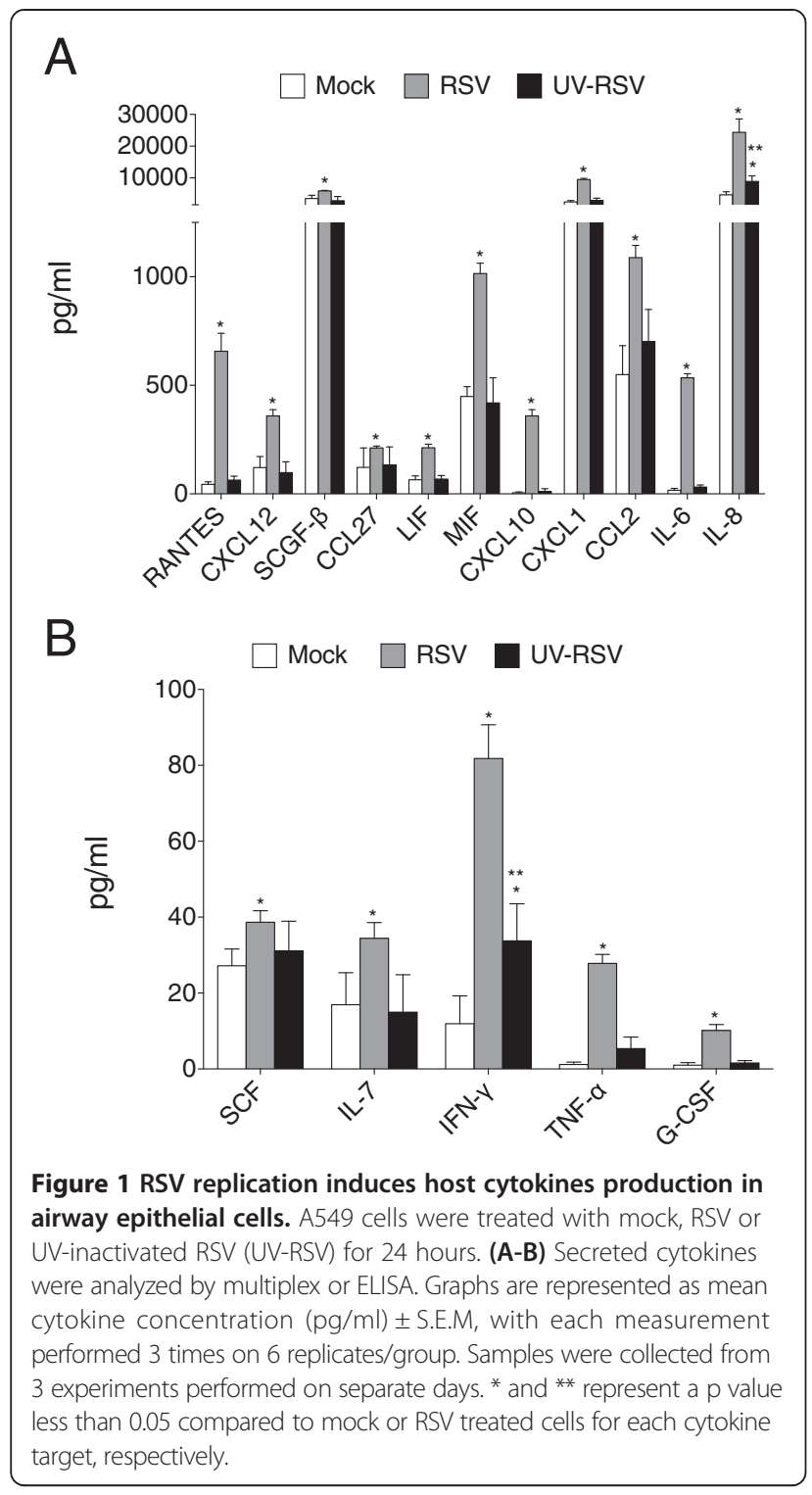

MIF, LIF, CCL27, CXCL12/SDF-1 $\alpha$, SCGF- $\beta$ and SCF are expressed in the airways of mice following RSV challenge Gene expression and BALF protein levels for MIF, LIF, CCL27, CXCL12/SDF-1 $\alpha$, SCGF- $\beta$ and SCF were examined in wild-type (FVB/NJ) mice $0,1,3,5,7$, and 9 days post mock, RSV or UV-RSV intranasal challenge (Figure 2), as these cytokines have not been reported in the literature to be RSV inducible. Increased gene expression of MIF, LIF, CXCL12, SCGF- $\beta$ and SCF was observed following 1 day of RSV challenge (Figure 2A). RSV inducible CCL27 was only observed 5 days post challenge. UV-inactivated RSV did not alter airway expression of these cytokines (Figure 2A). Multiplex or ELISA was performed to examine BALF protein levels of LIF, CCL27, CXCL12/SDF-1 $\alpha$, and SCF. Similarly to gene expression profiles, BALF levels of SCF, LIF, CCL27/CTAK and CXCL12/SDF-1 $\alpha$ were all enhanced in mice exposed to RSV infection compared to mock or UV-RSV treated animals (Figure 2B). These new RSV-inducible targets represent new plausible targets for investigation in the human disease.

RSV activates TLRs and RIG-I-like receptors (RLR)

Nasopharyngeal cells from infants with RSV infection have gene expression differences for MDA-5, RIG-1, TLR-7, and TLR-8 [23]. Since RSV infection induces a large repertoire of host cytokines, chemokines and growth factors, several of the major pathogen recognition receptors were profiled in each cell type to determine the potential regulators of the immune response to RSV infection. Therefore, qPCR and immunoblots were performed for p-IRF3(ser396), IRF3, p-TBK1(ser172), TBK-1/NAK, RIG-I, LGP2, MDA5, MAVS, LGP2, TLR3, TLR7, TLR8, TLR9, Trif, MyD88, IRAK1, NOD2 and $\beta$-actin. The remaining TLR genes and other NOD genes (NOD1 and NLXR1) were also profiled in both cell types. A549 and SAE cells became readily infected with RSV 24 hours after incubation (Figure 3A) and had significant increases in RIG-I, MDA5, LGP2, NOD2, Trif, TLR2 and TLR3 gene expression in both cell types (Figure 3B). TLR1, 6 and 10 gene expressions were also enhanced in SAE cells following RSV infection. TLR4 gene expression was increased in A549s cells. Protein profiles were analyzed for several of the receptors with the greatest gene induction with RSV infection, by immunoblots. Increased cellular protein levels of RIG-I, MDA5, LGP2, Trif and TLR3 were observed by immunblotting in both A549 and SAE cells (Figure 3C). Utilizing densitometry analysis, no significant changes in NOD2 and TLR2 protein levels were observed in either A549 or SAE cells possibility due to lower induction of gene expression for these targets (Additional file 1: Figure S2). To investigate the activation status of receptors, downstream signaling transduction analysis was performed for the phosphorylation of IRF3, TBK1, degradation of IRAK1 and increased expression of Trif and MyD88. IRF3 and TBK1 underwent phosphorylation following RSV infection, confirming activation of these pathways (Figure $3 \mathrm{C}$ and Additional file 1: Figure S2). Equally, increases in Trif and decreased IRAK1 levels were observed after RSV challenge (Figure 3C and Additional file 1: Figure S2), further confirming that several receptors underwent activation. Due to the activation intensity of the RLR, activation of IRF3 and induction of the Trif/TLR3, in vivo studies were undertaken specifically examining the Trif and RLR pathways.

\section{Trif and Mavs KO mice have reduced cytokine responses to RSV challenge}

Others have reported that RIG-I is essential for host immune defenses against RSV [30,31] and our in vitro 


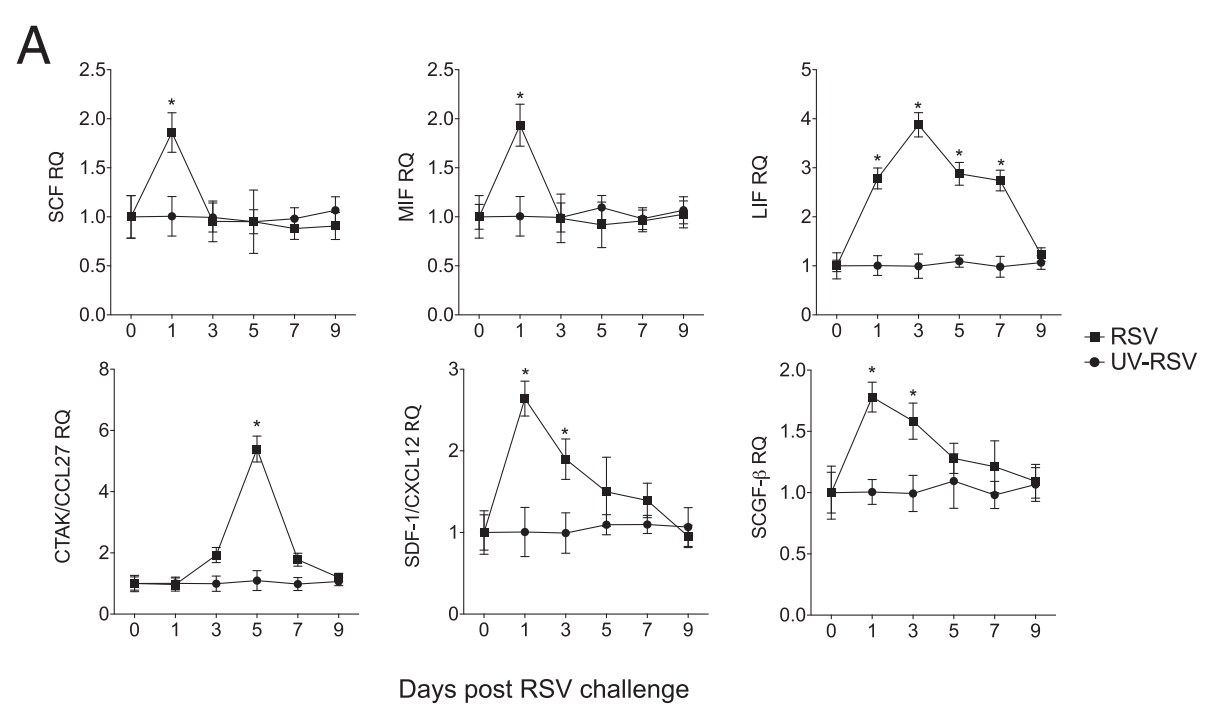

B
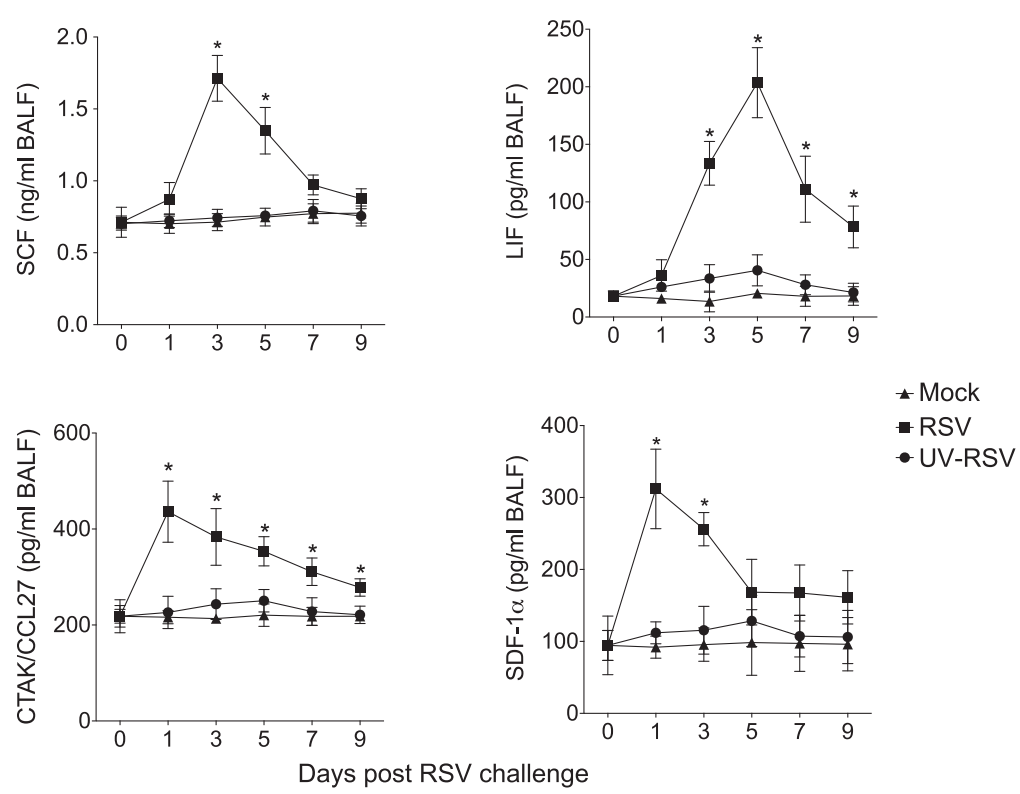

Figure 2 RSV challenge induces MIF, LIF, CCL27, CXCL12, SCGF- $\beta$ and SCF airway expression. FVB/NJ mice were infected with $1 \times 10^{6}$ pfu of RSV, UV-RSV or mock and animals were euthanized on days 0, 1, 3, 5, 7 and 9-post challenge. MIF, LIF, CTAK/CCL27, SDF-1/CXCL12, SCGF- $\beta$ and SCF (A) lung gene expression and (B) BALF protein levels were analyzed by qPCR and multiplex, respectively. Graphs are represented as relative quantification (RQ) or BALF concentration of the mean \pm S.E.M, with each measurement performed 3 times on 10 animals/group. *Represents a $p$ value less than 0.05 compared to mock treated mice on each corresponding day.

studies suggest that both TLR3 and RLR signaling are highly responsive to RSV. To assess the importance of RSV-induced TLR3 and RLR signaling on cytokine production, wild-type (FVB/NJ), Trif and Mavs KO mice were infected with RSV and cytokines levels were investigated by $\mathrm{qPCR}$ on lung tissue and multiplex analysis on BALF. Mavs KO mice were utilized as MAVS links RIG-I and MDA5 to antiviral effectors responses. Mice were exposed to RSV and euthanized days 1, 3, 5, 7 and 9 days after the RSV challenge. Loss of Trif and Mavs expression in mice did not significantly alter loss in body weight following RSV infection compared to wild-type mice (Figure 4A) but loss of Mavs or Trif resulted in a greater viral load in the airway tissue on day 9 following infection compared to wild-type mice (Figure 4B).

Cytokine release into the airways was assessed by multiplex analysis 3 days post RSV challenge and RSV infection yielded a significant release of IL- $1 \alpha$, IL- $1 \beta$, IL-2, IL-4, IL-5, IL-6, IL-12(p40), IL-12(p70), IL-13, IL-15, IFN- $\gamma$, MCP-1/CCL2, CCL5, CCL3, CCL4, CCL11, 


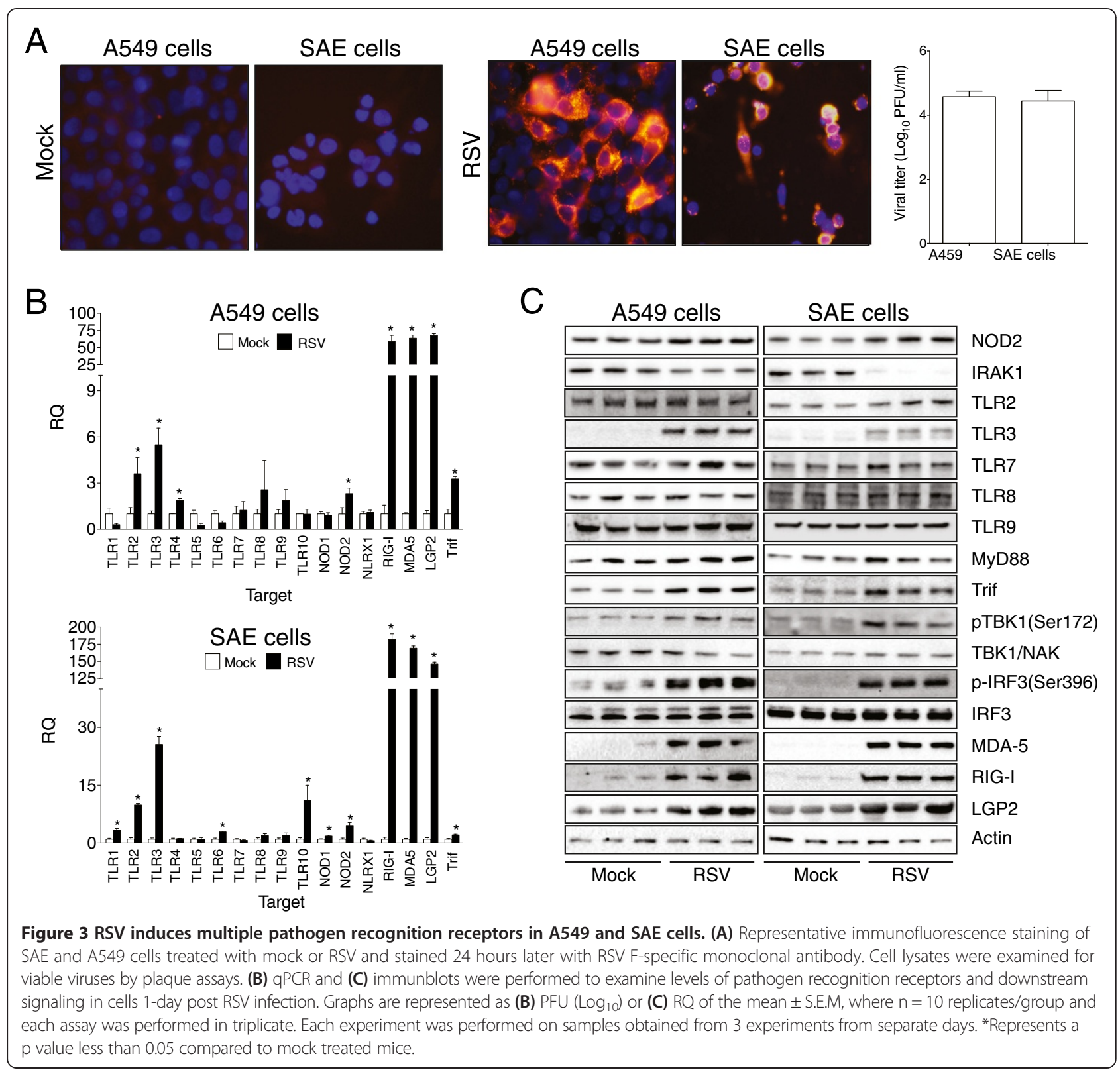

LIF, CTAK/CCL27, SDF-1 $\alpha /$ CXCL12, SCF and CXCL1 into the BALF of control mice (Table 2). RSV also increased gene expression of IL-27, CXCL9, CXCL10, CXCL11, MIF and SCGF- $\beta$ in the airways of control mice 1-day post RSV challenge (Table 3). Loss of Mavs expression subdued RSV-induced IL-1 $\beta$, IL-4, IL-5, IL-6, IL-12(p40), IFN- $\gamma$, CCL2, CCL3, CCL5 and CXCL1 release into the airways (Table 2). Mavs $\mathrm{KO}$ mice also had significantly reduced IL-22, CXCL9, CXCL10 and MIF gene expression compared to wild-type mice (Table 3). Loss of Trif expression altered the RSV induced release of IL-1 $\beta$, IL-5, CXCL12 and IFN- $\gamma$ (Table 2) and airway gene expression of MIF, LIF and CXCL12 (Table 2).
Silencing RIG-I expression in A549 cells subdues cytokine production

RLRs (RIG-I, MDA5 and LGP2) and Trif were silenced in A549 cells to establish which RLR signaling was required for RSV inducible host cytokines (See Additional file 1: Figure S3 for RLR and Trif knockdown). Loss of RIG-I expression significantly decreased RSV induced IL-1 $\beta$, IL-6, IL-7, IL-12(p70), MCP-1/CCL2, IP-9/ CXCL11, IP-10/CXCL10, TNF- $\alpha$, MIF, RANTES/CCL5 and SCGF- $\beta$ (Figure 5). Interestingly, SCGF- $\beta$ release was altered following loss of expression of RIG-I, MDA5 and LGP2 (Figure 5A). CTAK/CCL27 release was only altered following silencing of MDA5 or Trif expression (Figure 5A). 

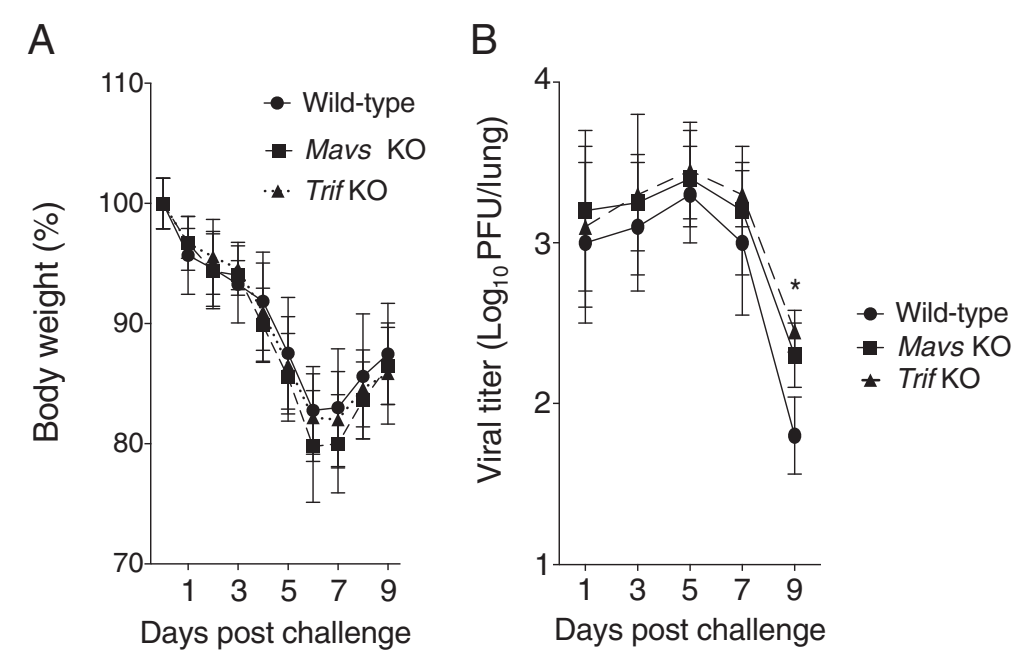

Figure 4 Loss of Trif or Mavs expression enhances RSV viral titer in the lungs of mice. (A) RSV infection resulted in a drop in body weight in all animal groups and (B) a significant increase in the viral load 9 days post RSV challenge in the Mavs KO mouse group compared to mock treated mice. Graphs are represented as mean \pm S.E.M., where each measurement was performed 3 times on 10 animals/group. *Represents a $\mathrm{p}$ value less than 0.05 comparing Mavs KO mice to wild-type mice 9 days post RSV challenge.

Trif expression regulated MIF, LIF, CTAK/CCL27, SDF$1 \alpha /$ CXCL12 and IL-1 $\beta$ (Figure 5). Therefore, these results show that host RIG-I is the primary RLR to regulate RSVmediated immune responses.

\section{Neutralization LIF enhances lung injury during an RSV infection}

Administration of recombinant LIF to the airways has a protective effect in response to LPS treatment [32], hyperoxia [33] and to bacterial pneumonia [29]. The functional relevance of LIF expression during a RSV infection is unknown. Administration of anti-LIF IgG resulted in RSV-infected animals losing weight faster than control IgG treated littermates (Figure 6A). The total number of immune cells in the BALF was comparable between RSV treated groups (Figure 6B). However, BALF cells in the animals administered anti-LIF IgG were undergoing apoptosis at a greater rate than control IgG animals (Figure 6B). Inhibition of LIF signaling also subdued RSV induced airway hyperresponsiveness, demonstrated by respiratory system resistance (Rn) measurements during a methacholine dose challenge (Figure 6C). Anti-LIF IgG treated mice had significantly higher protein concentration (Figure 6D) in the BALF compared to infected control IgG treated mice, suggesting potentially increased damage and disruption of the epithelial cell barrier. Lung RSV burdens were not significantly affected by LIF blockade seven days following RSV challenge (Figure 6E). Lung cytokine gene expression was determined by $\mathrm{qPCR}$ and blocking LIF signaling resulted in enhanced CXCL1, RANTES/ CCL5, CXCL10/IP-10, MIP-1 $\alpha /$ CCL3 and MCP-1/CCL2 compared to control IgG treated mice, 7-days post RSV infection (Figure 6F). Altogether, these results suggest that LIF expression suppresses lung cell apoptosis, airway hyperresponsiveness, epithelial cell barrier damage, cytokine production and subsequently airway injury.

\section{Discussion}

To our knowledge, these findings represent the first evidence that MIF, LIF, CTAK/CCL27, SDF-1 $\alpha /$ CXCL12, SCGF- $\beta$ and SCF are expressed during an RSV infection and LIF signaling is critical to prevent lung damage. Others and we demonstrate that a spectrum of cytokines is released to counter RSV infection [1,7-10,34] but further analysis is required to determine the role of these immune responses in RSV pathology. The results of this study demonstrate that RLRs and Trif signaling pathways play major roles in the immune response to RSV infection for both inflammation induction and resolution. Interestingly, 34 of the 42 cytokines examined in A549 and SAE cells were released upon RSV stimulation. This study highlights the robust epithelial responses to RSV infection, characterizes two major signaling cascade needed to counter viral infectivity and identified the role of LIF signaling during an RSV infection.

Many of the RSV-inducible cytokines described in this study have already been described in clinical samples, in vitro epithelial cell culture or in animals models [1,7-16], such as elevated MIG/CXCL9 levels in BALB/c mice inoculated with RSV [35]. Importantly, increases in media or mouse BALF IL1-RA, IL-6, IL-7, CXCL10/ IP-10, CXCL8/IL-8, CCL2/MCP-1, CCL3/MIP-1 $\alpha$, CCL4/ MIP-1 $\beta$, TNF- $\alpha$, IFN- $\gamma$ and CCL5/RANTES following 
Table 2 RSV infections induce airway cytokine release in mice

\begin{tabular}{|c|c|c|c|c|c|c|}
\hline \multirow[b]{2}{*}{ Cytokine (pg/ml BALF) } & \multicolumn{2}{|l|}{ Wild-type } & \multicolumn{2}{|l|}{ Trif KO } & \multicolumn{2}{|l|}{ Mavs KO } \\
\hline & Mock & RSV & Mock & RSV & Mock & RSV \\
\hline IL-1a & $3.54 \pm 1.51$ & $30.11 \pm 9.64^{*}$ & $9.62 \pm 8.57$ & $25.53 \pm 5.33$ & $6.74 \pm 3.56$ & $24.55 \pm 3.36$ \\
\hline IL-1 $\beta$ & $66.61 \pm 5.52$ & $78.55 \pm 6.66^{*}$ & $67.05 \pm 6.19$ & $66.16 \pm 5.00^{*}$ & $56.88 \pm 2.83$ & $49.18 \pm 4.06^{\#}$ \\
\hline IL-2 & $7.25 \pm 0.32$ & $5.52 \pm 0.31 *$ & $6.44 \pm 0.03$ & $5.91 \pm 1.22$ & $5.36 \pm 0.35$ & $6.91 \pm 0.75$ \\
\hline IL-3 & $29.39 \pm 1.54$ & $28.87 \pm 0.84$ & $30.50 \pm 1.55$ & $28.95 \pm 1.47$ & $22.98 \pm 2.89$ & $24.37 \pm 0.51$ \\
\hline IL-4 & $15.00 \pm 0.01$ & $48.12 \pm 0.02^{*}$ & $17.21 \pm 0.01$ & $32.89 \pm 0.01$ & $14.41 \pm 0.01$ & $16.33 \pm 0.01^{\#}$ \\
\hline IL-5 & $4.15 \pm 0.27$ & $20.00 \pm 5.42^{*}$ & $5.39 \pm 0.63$ & $7.55 \pm 1.46^{\#}$ & $4.37 \pm 0.15$ & $4.81 \pm 0.50^{\#}$ \\
\hline IL-6 & $3.78 \pm 0.44$ & $9.63 \pm 3.80^{*}$ & $4.19 \pm 0.57$ & $9.79 \pm 3.16$ & $3.18 \pm 0.31$ & $5.55 \pm 1.16^{\#}$ \\
\hline IL-9 & $204.10 \pm 11.28$ & $233.11 \pm 18.25$ & $230.31 \pm 37.74$ & $216.01 \pm 18.58$ & $181.42 \pm 12.58$ & $228.93 \pm 19.01$ \\
\hline IL-10 & $61.43 \pm 9.92$ & $76.45 \pm 22.60$ & $46.08 \pm 8.18$ & $166.2 \pm 55.5$ & $51.13 \pm 8.32$ & $63.33 \pm 12.23$ \\
\hline IL-12(p40) & $8.69 \pm 0.17$ & $11.95 \pm 1.65^{*}$ & $9.95 \pm 0.97$ & $10.99 \pm 1.18$ & $6.89 \pm 1.06$ & $8.74 \pm 0.28^{\#}$ \\
\hline IL-12(p70) & $11.34 \pm 0.98$ & $10.23 \pm 1.43$ & $13.14 \pm 3.60$ & $14.89 \pm 2.85$ & $8.60 \pm 2.10$ & $12.01 \pm 3.70$ \\
\hline IL-13 & $48.41 \pm 2.44$ & $29.61 \pm 3.50^{*}$ & $41.84 \pm 4.94$ & $26.51 \pm 2.15$ & $47.99 \pm 2.33$ & $30.31 \pm 6.52$ \\
\hline IL-15 & $6.96 \pm 1.09$ & $14.25 \pm 3.31^{*}$ & $13.46 \pm 3.62$ & $9.83 \pm 1.89$ & $7.42 \pm 2.18$ & $10.83 \pm 1.68$ \\
\hline IL-17 & $43.66 \pm 5.10$ & $64.17 \pm 16.72$ & $37.61 \pm 30.20$ & $79.81 \pm 13.48$ & $41.34 \pm 5.01$ & $55.44 \pm 4.68$ \\
\hline IFN- $\nu$ & $12.15 \pm 0.76$ & $35.47 \pm 11.26^{*}$ & $10.19 \pm 1.26$ & $8.24 \pm 0.61^{\#}$ & $11.74 \pm 5.04$ & $13.22 \pm 3.78^{\#}$ \\
\hline $\mathrm{MCP}-1 / \mathrm{CCL} 2$ & $64.57 \pm 2.12$ & $85.14 \pm 8.23^{*}$ & $67.87 \pm 5.15$ & $81.61 \pm 7.86$ & $63.22 \pm 5.01$ & $69.99 \pm 4.82^{\#}$ \\
\hline RANTES/CCL5 & $12.41 \pm 0.84$ & $107.32 \pm 21.70^{*}$ & $18.02 \pm 3.32$ & $128.81 \pm 23.27$ & $15.01 \pm 0.98$ & $68.32 \pm 11.90^{\#}$ \\
\hline TNF- $a$ & $524.98 \pm 41.34$ & $438.88 \pm 55.48$ & $602.71 \pm 38.15$ & $460.01 \pm 84.64$ & $504.18 \pm 32.14$ & $448.88 \pm 34.48$ \\
\hline MIP-1a/CCL3 & $105.01 \pm 1.38$ & $164.72 \pm 5.56^{*}$ & $123.02 \pm 18.29$ & $132.58 \pm 12.77$ & $95.96 \pm 4.84$ & $106.61 \pm 4.60^{\#}$ \\
\hline MIP-1 $\beta / C C L 4$ & $66.37 \pm 2.89$ & $112.91 \pm 19.50^{*}$ & $49.59 \pm 4.74$ & $103.10 \pm 18.43$ & $54.40 \pm 10.08$ & $127.45 \pm 41.05$ \\
\hline MIP-2/CXCL2 & $10.22 \pm 0.56$ & $10.82 \pm 2.04$ & $14.86 \pm 1.86$ & $13.40 \pm 2.47$ & $9.15 \pm 0.80$ & $13.00 \pm 1.78$ \\
\hline Eotaxin/CCL11 & $5.72 \pm 0.55$ & $25.02 \pm 5.97^{*}$ & $9.26 \pm 2.68$ & $15.37 \pm 3.80$ & $5.94 \pm 0.50$ & $13.62 \pm 3.08$ \\
\hline GM-CSF/CSF2 & $41.63 \pm 3.79$ & $45.57 \pm 3.25$ & $49.61 \pm 3.60$ & $57.88 \pm 6.41$ & $37.14 \pm 6.15$ & $45.93 \pm 2.41$ \\
\hline M-CSF/CSF1 & $24.93 \pm 2.08$ & $26.73 \pm 2.47$ & $29.13 \pm 2.97$ & $28.85 \pm 3.79$ & $20.40 \pm 1.97$ & $25.94 \pm 2.84$ \\
\hline LIF & $12.37 \pm 5.4$ & $70.18 \pm 12.33^{*}$ & $11.12 \pm 2.12$ & $60.42 \pm 10.32$ & $12.91 \pm 3.99$ & $72.32 \pm 7.69$ \\
\hline CTAK/CCL27 & $231 \pm 21.23$ & $430 \pm 43.21 *$ & $220 \pm 18.91$ & $389.1 \pm 21.98$ & $198.3 \pm 10.99$ & $424.7 \pm 34.76$ \\
\hline SDF-1a/CXCL12 & $94.0 \pm 10.16$ & $312.2 \pm 50.96^{*}$ & $51.1 \pm 23.23$ & $77.21 \pm 13.11^{\#}$ & $110.21 \pm 20.20$ & $296.19 \pm 15.07$ \\
\hline SCF & $711.00 \pm 40.15$ & $1713.06 \pm 98.19^{*}$ & $721.79 \pm 88.95$ & $1392.90 \pm 99.41$ & $702.83 \pm 76.41$ & $1721.22 \pm 70.60$ \\
\hline GROa/KC/CXCL1 & $1.02 \pm 0.45$ & $81.35 \pm 16.70$ & $1.24 \pm 0.16$ & $81.29 \pm 23.27$ & $4.35 \pm 0.97$ & $48.55 \pm 20.84^{\#}$ \\
\hline
\end{tabular}

Cytokine levels were determined in BALF from wild-type, Trif KO and Mavs KO mice 3 days post RSV challenge, by multiplex analysis. Values are represented as mean BALF concentration $(\mathrm{pg} / \mathrm{ml}) \pm$ S.E.M. Each assay was performed in triplicate, where $\mathrm{n}=10$ animals/group. Bold numbers denoted by *represents a $\mathrm{p}$ value less than 0.05 compared to mock treated mice. "denotes a p value less than 0.05 compared to RSV treated wild-type mice.

RSV infection reflects similar observations from clinical studies $[10,36]$. To our knowledge, we are the first to identify that RSV infection in epithelial cells and mice can induce MIF, LIF, CTAK/CCL27, SDF- $1 \alpha /$ CXCL12, SCGF- $\beta$ and SCF expression and secretions. This is an important finding as each new target can potentially play a different role in RSV clearance. MIF activates lymphocytes, granulocytes and monocytes/macrophages and plays a key role in several host immune cell responses [37]. CTAK/CCL27 is a cytokine associated with $\mathrm{T}$ cell activation and migration [38] and is elevated in severe tuberculosis cases [39]. Recombinant protein SDF-1 $\alpha /$ CXCL12 does not prevent RSV infection of Hep2 cells [40] but SDF-1 $\alpha /$ CXCL12 is strongly chemotactic for lymphocytes [41] and contributes to pulmonary fibrosis [42]. The potential role of SCGF- $\beta$ in RSV infections is not as straightforward to elucidate but SCGF- $\beta$ can support growth of primitive hematopoietic cells [43] and can promotes proliferation of erythroid or myeloid progenitors. SCF and its soluble receptor c-kit correlate with asthma severity [44]. This study identified an important role for LIF in RSV infection. Therefore, identifying these new RSV inducible targets in clinical samples and their role in viral clearance may provide important insights into host responses to RSV infection.

Other investigators have demonstrated the therapeutic potential of LIF expression in other models of 
Table 3 RSV infections enhance airway cytokine gene expression

\begin{tabular}{|c|c|c|c|c|c|c|}
\hline \multirow[b]{2}{*}{ Cytokine (pg/ml) } & \multicolumn{2}{|l|}{ Wild-type } & \multicolumn{2}{|l|}{ Trif KO } & \multicolumn{2}{|l|}{ Mavs KO } \\
\hline & Mock & RSV & Mock & RSV & Mock & RSV \\
\hline$\overline{I L}-4$ & $1.00 \pm 0.40$ & $4.67 \pm 1.26^{*}$ & $1.26 \pm 0.33$ & $3.97 \pm 0.53$ & $0.27 \pm 0.14$ & $0.23 \pm 0.10^{\#}$ \\
\hline IL-6 & $1.00 \pm 0.10$ & $4.15 \pm 0.14^{*}$ & $1.30 \pm 0.33$ & $3.32 \pm 0.52$ & $1.81 \pm 0.71$ & $1.61 \pm 0.57^{\#}$ \\
\hline IL-18 & $1.00 \pm 0.19$ & $0.71 \pm 0.16$ & $0.64 \pm 0.15$ & $0.43 \pm 0.03$ & $0.51 \pm 0.06$ & $0.60 \pm 0.34$ \\
\hline IL-22 & $1.00 \pm 0.41$ & $1.19 \pm 0.16$ & $0.98 \pm 0.07$ & $1.49 \pm 0.49$ & $0.54 \pm 0.30$ & $0.45 \pm 0.08^{\#}$ \\
\hline IL-23 & $1.00 \pm 0.41$ & $1.48 \pm 0.27$ & $0.75 \pm 0.17$ & $1.41 \pm 0.20$ & $0.19 \pm 0.15$ & $1.03 \pm 0.02$ \\
\hline$\| \mathrm{L}-27$ & $1.00 \pm 0.15$ & $2.00 \pm 0.10^{*}$ & $0.98 \pm 0.31$ & $1.88 \pm 0.39$ & $0.49 \pm 0.06$ & $1.63 \pm 0.09$ \\
\hline CXCL1/KC & $1.00 \pm 0.12$ & $11.91 \pm 0.05^{*}$ & $1.56 \pm 0.67$ & $12.04 \pm 0.37$ & $0.29 \pm 0.11$ & $3.23 \pm 0.12^{\#}$ \\
\hline MIG/CXCL9 & $1.00 \pm 0.58$ & $160.81 \pm 17.67^{*}$ & $3.18 \pm 1.10$ & $148.83 \pm 16.68$ & $0.23 \pm 0.14$ & $29.47 \pm 5.42^{\#}$ \\
\hline IP10/CXCL10 & $1.00 \pm 0.18$ & $8.07 \pm 1.62^{*}$ & $1.78 \pm 0.43$ & $9.77 \pm 2.51$ & $0.312 \pm 0.10$ & $3.59 \pm 2.07^{\#}$ \\
\hline IP9/CXCL11 & $1.00 \pm 0.12$ & $2.61 \pm 0.26^{*}$ & $1.30 \pm 0.44$ & $4.38 \pm 0.85$ & $0.18 \pm 0.16$ & $3.19 \pm 1.05$ \\
\hline MIF & $1.00 \pm 0.19$ & $2.40 \pm 0.26^{*}$ & $1.09 \pm 0.15$ & $0.90 \pm 0.41^{\#}$ & $0.93 \pm 0.11$ & $0.52 \pm 0.40^{\#}$ \\
\hline LIF & $1.00 \pm 0.14$ & $2.71 \pm 0.09 *$ & $1.16 \pm 0.66$ & $1.01 \pm 0.27^{\#}$ & $0.99 \pm 0.11$ & $2.43 \pm 0.13$ \\
\hline CTAK/CCL27 & $1.00 \pm 0.28$ & $5.79 \pm 0.67^{*}$ & $1.18 \pm 0.18$ & $4.23 \pm 0.68$ & $0.73 \pm 0.18$ & $4.27 \pm 0.42$ \\
\hline SDF-1a/CXCL12 & $1.00 \pm 0.16$ & $2.67 \pm 0.96^{*}$ & $1.28 \pm 0.23$ & $1.57 \pm 0.51^{\#}$ & $0.51 \pm 0.20$ & $2.59 \pm 1.07$ \\
\hline SCGF- $\beta$ & $1.00 \pm 0.22$ & $1.87 \pm 0.16^{*}$ & $1.33 \pm 0.14$ & $1.88 \pm 0.81$ & $0.88 \pm 0.26$ & $1.19 \pm 0.15$ \\
\hline SCF & $1.00 \pm 0.15$ & $1.60 \pm 0.16^{*}$ & $1.09 \pm 0.25$ & $1.20 \pm 0.41$ & $0.93 \pm 0.11$ & $1.82 \pm 0.40$ \\
\hline TGF- $\beta$ & $1.00 \pm 0.16$ & $1.47 \pm 0.06$ & $0.84 \pm 0.19$ & $1.00 \pm 0.43$ & $0.51 \pm 0.01$ & $0.62 \pm 0.43$ \\
\hline MCP-1/CCL2 & $1.00 \pm 0.08$ & $12.39 \pm 3.01 *$ & $2.38 \pm 0.73$ & $20.45 \pm 4.31$ & $0.45 \pm 0.07$ & $5.13 \pm 1.33^{\#}$ \\
\hline MIP-1a/CCL3 & $1.00 \pm 0.08$ & $10.17 \pm 1.51^{*}$ & $1.68 \pm 0.40$ & $13.64 \pm 1.51$ & $0.58 \pm 0.14$ & $3.37 \pm 0.83^{\#}$ \\
\hline
\end{tabular}

Cytokine gene expression levels were determined in lung tissue from wild-type, Trif KO and Mavs KO mice 1 days post RSV challenge by qPCR, except CTAK/ CCL27 which was examined 5 days post RSV challenge. Values are represented as mean RQ (relative to mock treated animals, using $\beta$-Actin as an endogenous control.) \pm S.E.M. Each assay was performed in triplicate, where $n=10$ animals/group. Bold numbers denoted by *represents a $p$ value less than 0.05 compared to mock treated mice. "denotes a p value less than 0.05 compared to RSV treated wild-type mice.

lung injury, as it protects against lung injury [29,32,33]. Recombinant LIF reduces cytokine expression [29] and alters alveolar neutrophil numbers [29,32]. Lif $\mathrm{KO}$ animals have increased expression of CXCL1, GM-CSF, RANTES/CCL5 and MIP-1 $\alpha /$ CCL3 early during an experimental autoimmune encephalomyelitis model and reduced CCL2, CCL3, and CXCL10 at a later stage of the disease [45]. In this study neutralizing LIF signaling enhanced CXCL1, RANTES/CCL5, CXCL10/IP-10, MIP$1 \alpha / C C L 3$ and MCP-1/CCL2 at day 7 of an RSV infection. Therefore LIF expression has the potential to regulate key factors in the immune system. Targeted overexpression of LIF in mouse airway epithelial cells significantly protects the airways during hyperoxia, with improved survival and decreased pulmonary edema [33]. Other IL-6 family members (IL-6 [46] and IL-11 [47]) have similar functions, which suggest that IL-6 family cytokines may collectively protect lungs from injury. LIF is a prominent STAT3-activating cytokine that facilitates tissue protection during pneumonia [29] and plays a similar airway protective role during RSV infection. The significance of LIF expression and STAT3 activation in RSV infection represents an intriguing area for further study. LIF has been shown to regulate apoptosis with some investigators suggesting LIF acts as a pro-apoptotic mediator $[48,49]$ while others have demonstrated LIF to have anti-apoptotic potential $[50,51]$. In this study increased apoptotic BAL cells were observed in animals administered anti-LIF IgG prior to RSV infection. Enhanced Fas, Fap, Il24, and Tnfsf15 expression is observed following LIF depletion in animals with bacterial pneumonia [29], which could contribute to the increased apoptosis observed here. Whether this induction of apoptosis contributes to the pathology observed in this study is unknown and requires further investigation. Epithelial damage correlates with airway hyperreactivity in asthma patients [52] and the increased protein levels in the BALF of animals treated with anti-LIF IgG prior to RSV infection could also contribute to the enhanced airway resistance observed in these animals. LIF enhances the maintenance of stem cells [27], which may directly impact lung tissue regeneration and repair [53], similar to IL-6 activation of STAT3 [54]. These data reveal the vast biological processes of LIF signaling during RSV infection. Whether the other cytokines identified in this study also play such major roles on disease pathology needs to be addressed.

Our study also reveals that Trif and RLR signaling regulate a significant proportion of the cytokines induced 

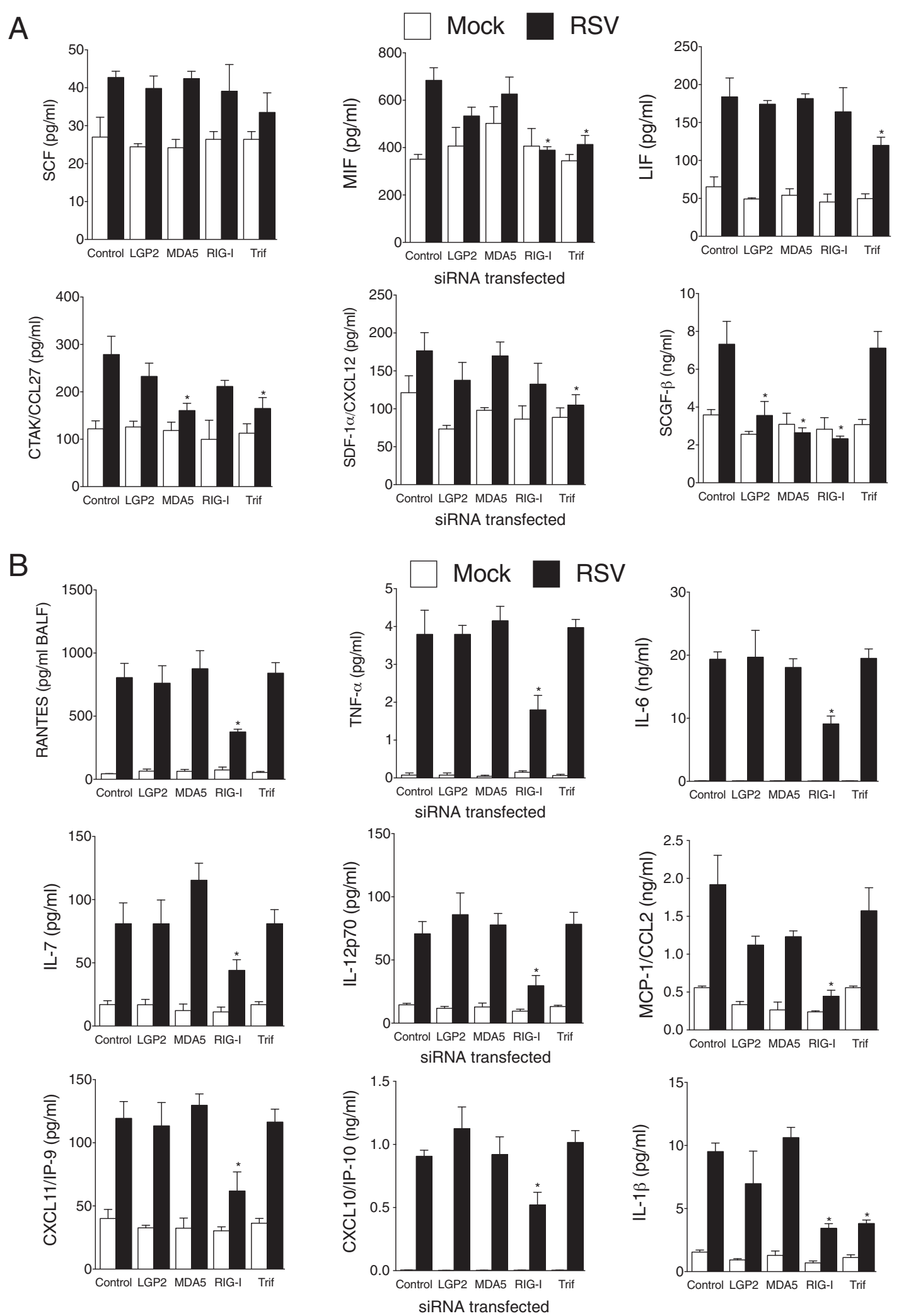

Figure $\mathbf{5}$ (See legend on next page.) 
(See figure on previous page.)

Figure 5 Silencing RIG-I subdues RSV induced cytokine release from A549 cells. (A-B) Multiplex or ELISA analysis was performed on secreted cytokines from A549 cells transfected with siRNA targeting RIG-I (DDX58), LGP2 (DHX58), MDA5 (IFIH1), Trif or a negative sequence control. Graphs are represented as mean cytokine concentration \pm S.E.M, where $n=10$ replicates/group and each assay were performed in triplicate. Each experiment was performed on samples obtained from 3 experiments from separate days. *Represents a p value less than 0.05 compared to control siRNA treated cells following RSV infection.

by RSV infection, with LIF expression sensitive to Trif activation. Prior to undertaking this study, several pathogen recognition receptors were associated with regulating the immune response against RSV infection, with RIG-I [55], TLR7 [56] and TLR3 [57] known to affect numerous cytokine responses. Several investigators characterized the RIG-I-like family proteins as critical for the detection of RSV, using human cell lines [30] and knockout mice [58]. RIG-I principally recognizes dsRNA [59]; however Sendai virus defective interfering RNA [60] and the genomic "panhandle" structure of influenza virus [61] activate RLR signaling. These "panhandle" structures allow negative-strand RNA viral genomes to achieve partially double-stranded RNA [62], which may allow RSV to induce RLRs. Our study observed early cytokine responses following RSV infection and identified a pronounced RIG-I response, which could be lost over time following NS1 or NS2 binding to RIG-I/ MDA5 as reported by others $[63,64]$. Also, RSV induction of TLR3 is regulated by RIG-I-dependent IFN- $\beta$ secretions from infected epithelial cells, which is mediated by both IFN response-stimulated element (ISRE) and signal transducer and activator of transcription (STAT) sites in its proximal promoter [65]. Therefore there are multiple levels of RLRs regulation in RSV infections that can both trigger RLRs responses or subsequently adapt to block RLR-mediated signaling.

Recently, several investigators have utilized welldefined primary cell culture techniques to investigate viral-host interactions $[1,66,67]$, which may be a closer representation of human RSV infections compared to the approach we employed here. IFN- $\alpha / \beta$ are not secreted by RSV-infected well-defined bronchial epithelial cells [66], suggesting that there could be major signaling differences in well-defined primary cell culture techniques and monolayers. However, our overall findings suggest that utilizing cell monolayers in combination with mouse models is an acceptable approach as our cytokine profile are comparable to that observed in the human disease state [10]. In addition, our study has exposed new RSV inducible host immune response cytokines. Since we only explored epithelial cell responses in vitro, our in vivo parallel approach also validates our monolayer results and suggests that the epithelium is a significant source of initial immune responses following RSV infection.

\section{Conclusions}

Our studies demonstrate that RSV exposure stimulates a cascade of early immune response in epithelial cells and a large number of these responses act in an RLR/Trif dependent manner. These findings also identified new cytokines that are triggered by RSV lung infection, with LIF signaling critical for the protection of the lung from injury during RSV infection. Profiling the loss and gain of the other identified cytokines (MIF, CTAK/CCL27, SDF- $1 \alpha /$ CXCL12, SCGF- $\beta$ and SCF) over the course of an RSV infection may help to decipher the relevancy of these cytokines in viral clearance and lung integrity, and represents important questions to be addressed by future studies.

\section{Methods}

\section{RSV culture}

Human RSV strain A2 (ATCC, Manassas, VA; \#VR1540) was infected at a multiplicity of 0.1 into Hep2 cells. The virus was allowed to grow for 5 days at $37^{\circ} \mathrm{C}$ in a $5 \% \mathrm{CO}_{2}$ atmosphere. The infected Hep2 monolayers were collected and the virus was released by sonication. Cell debris was removed by centrifugation at $2500 \times g$ for 5 minutes at $4^{\circ} \mathrm{C}$. Virus was collected by centrifuging the supernatant for 2 hours at $22000 \times g$ at $4^{\circ} \mathrm{C}$. Virus were suspended in culture media and snap frozen and maintained at $-80^{\circ} \mathrm{C}$. Infectious virus titers were determined on Hep2 cells by performing serial dilution of the RSV stocks and counting infected cells stained for indirect immunofluorescence with an RSV F-specific monoclonal antibody (Abcam, Cambridge, MA). Additionally, plaque assays were performed as previously described [68] on Hep2 cells using methyl cellulose overlay media and staining with $0.5 \mathrm{mg} / \mathrm{ml}$ thiazolyl blue tetrazolium bromide (MTT; Sigma Aldrich) solution for 3 hours at $37^{\circ} \mathrm{C}$. Non-infected cells were processed in the same manner as RSV infected cells and the resulting sample collection was used as a mock control. For experiments examining the effects of non-infectious RSV (UV-RSV), RSV preparations were UV-inactivated.

\section{Cell culture}

Monolayers of human SAE cells (Lonza, Walkersville, MD) from healthy subjects and A549 cells were cultured under submerged conditions. SAE cells were used for experiments at passages 3-6 and at a confluency of 


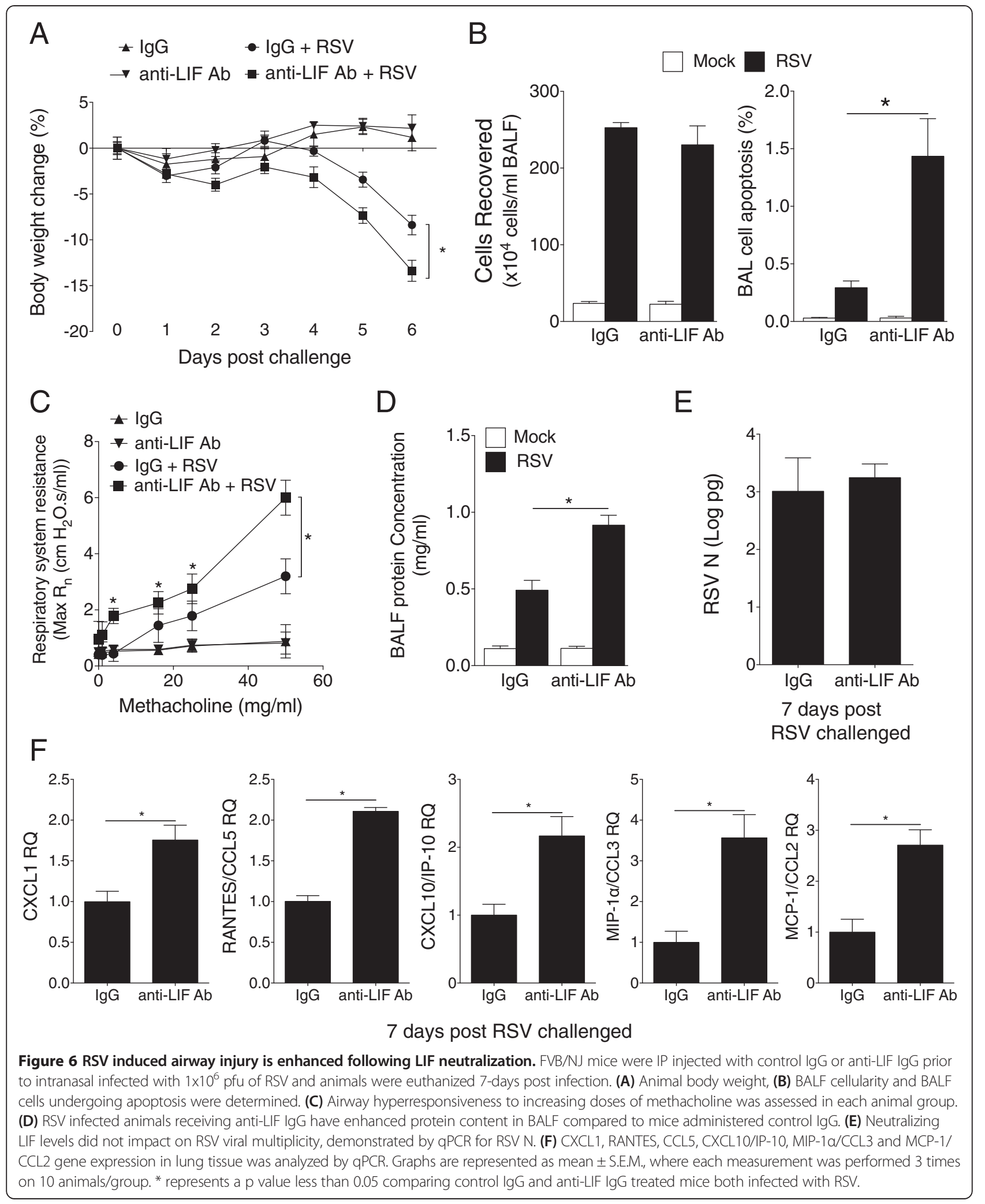

approximately $70 \%$. Cells were treated with RSV at a multiplicity of infection (MOI) of 0.3 for 24 hours. Immunoreactivity assays were performed on SAE and
A549 cells with polyclonal anti-RSV (Abcam; ab20745) antibodies. Cells were also treated with mock control, as described above. Additionally, A549 cells SAE cells were 
transfected by administering siRNA for MDA5, RIG-I, LGP2, Trif or control siRNA (Qiagen, Gaithersburg, MD).

\section{Animal models}

Trif (Ticam1) and Mavs knockout (KO) mice were purchased from the Jackson Laboratory (Bar Harbor, ME) and bred on to a FVB/NJ background at least 6 generations. All mice were maintained in a specific pathogenfree facility at Mount Sinai Roosevelt Hospital. 8-week-old mice were used at the initiation point for all experiments and each experimental parameter had 10 animals/group. Mice were anesthetized by intraperitoneal (IP) injection of a mixture of ketamine and xylazine. Animals were intranasally administered $1 \times 10^{6}$ PFU RSV or mock. Animals were euthanized on days $0,1,3,5,7$ and 9 post RSV or mock administering. Bronchoalveolar lavage fluid (BALF) and tissues were collected for cytokine analysis. $\mathrm{FVB} / \mathrm{NJ}$ mice were IP injected with $100 \mu \mathrm{g}$ of normal goat IgG (R\&D Systems) or a neutralizing goat polyclonal IgG targeting murine LIF (R\&D Systems) 2 hours prior to RSV administration. Lung viral titer and RSV N copy number were determined as previously described [69]. BALF cells were analyzed for apoptosis utilizing the LIVE/ DEAD cell viability assay from Life Technologies on the Guava easyCyte flow cytometer from Millipore. All animal experiments were performed with approval from Mount Sinai Roosevelt's Hospital's Institutional Animal Care and Use Committee approval.

\section{Cytokine measurements}

Mouse IL-4, IL-6, IL-18, IL-22, IL-23, IL-27, CXCL1/KC, CXCL9, CXCL10/IP-10, CXCL11, MIF, TGF- $\beta$, CCL2, LIF, CXCL12, CCL27, SCGF- $\beta$, SCF, MCP-1/CCL2, RANTES/CCL5 and MIP-1 $\alpha / C C L 3$ gene expression was performed by quantitative PCR (qPCR) using Taqman probes (Life technologies/Applied Biosystems, Carlsbad, CA). 42 human and 26 mouse cytokines were examined in human cell media and mouse BALF, respectively, using beads assays (Bio-Rad Magnetic Cytokine Bead Panels, groups 1 and 2) with the BioRad Bio-Plex 200 system (BioRad, Hercules, CA). ELISAs were utilized to determine mouse CXCL12 (R\&D systems), CCL27, SCGF- $\beta$ and SCF (Abcam) BALF levels.

\section{Intracellular signaling}

Cells were lysed in radio-immunoprecipitation assay (RIPA) buffer, centrifuged at $13,000 \times g$ for 10 minutes and supernatants collected. Immunoblots were conducted to determine levels of p-IRF3(ser396), IRF3, p-TBK1/NAK (Ser172), TBK1/NAK, RIG-I, MDA5, LGP2, TLR2, TLR3, TLR7, TLR8, TLR9, Trif, MyD88, IRAK1, NOD2 and actin (all antibodies from Cell Signaling Technologies). TLRs, NOD1, NOD2, NXLR1, RIG-I, MDA5, Trif and actin expressions were determined by qPCR using Taqman probes (Life technologies/Applied Biosystems).

\section{Airway responses to methacholine challenge}

Airway responses to methacholine (Sigma Chemical, St. Louis, MO) were assessed with the Scireq Flexivent system (Scireq, Montreal, QC, Canada) 1-week post RSV challenge and IP injection of control IgG or anti-LIF IgG. Animals were anesthetized with ketamine/xylazine $(10 \mathrm{mg} / \mathrm{kg})$ and paralysis was induced with $1 \mathrm{mg} / \mathrm{kg}$ pancuronium bromide IP (Sigma). The linear singlecompartment model was used to assess total respiratory system resistance $(\mathrm{Rn})$. Methacholine dose responses were determined.

\section{Statistical analyses}

For statistical analysis, data from 10 animals or multiple separate cell experiments were pooled. Data are expressed as means \pm S.E.M. Differences between groups of mice over time were compared by two-way analysis of variance (ANOVA). Individual differences between groups were tested by multiple comparison and analysis using the Bonferroni post-test. Pairs of groups were compared by Student's $\mathrm{t}$ test (two tailed). $\mathrm{p}$ values for significance were set at 0.05. All analysis was performed using GraphPad Prism Software (Version 5 for Mac OS X).

\section{Additional file}

Additional file 1: Figure S1. Profiling A549 cell cytokine response utilizing protein arrays. A549 cells were infected at an $\mathrm{MOI}$ of 0.3 for 24 hours. Cytokine arrays were performed with 24-hour media from mock and RSV treated cells. Densitometry were performed. Each pair of horizontal spots represents one cytokine. Graphs are represented as mean pixel intensity \pm S.E.M, where $n=2$ spots/group and each assay were performed on 3 samples/group collects on separate days. Figure S2. Quantification of pathogen recognition receptors and kinase immunoblots of RSV treated A549 and SAE cells. (A) SAE and (B) A549 cells were treated with mock or RSV (MOI of 0.3$)$ for 24 hours. Immunoblots (from Figure 3B) were subjected to densitometry analysis. Graphs are represented as mean pixel intensity (ratios of total protein to actin or phosphorylated protein to total protein) \pm S.E.M, where $n=3$ replicates/ group and each assay were performed on samples obtained on multiple days (3) and from multiple immunoblots. Figure S3. Confirmation that expression of Trif and RLRs was silenced in A549 cells. A549 cells were transfected with siRNA targeting RIG-I (DDX58), LGP2 (DHX58), MDA5 (IFIH1), Trif or a negative sequence control. Immunoblots were performed to determine RIG-I, LGP2, MDA5 and Trif protein levels compared to actin.

\section{Abbreviations}

RSV: Respiratory syncytial virus; BALF: Bronchoalveolar lavage fluid; SAE: Small airway epithelial; RIG-I: Retinoic acid-inducible gene-1; MDA5: Melanoma differentiation-associated protein-5; MAVS: Mitochondrial antiviral-signaling protein; RLRs: RIG-I-like receptors; NOD: Nucleotide-binding oligomerization domain-containing protein; LGP2: Laboratory of Genetics and Physiology 2; LIF: Leukemia inhibitory factor; MIF: Migration inhibitory factor; SCF: Stem cell factor (SCF); SCGF- $\beta$ : Stem cell growth factor beta.

\section{Competing interests}

The authors declare that they have no competing interests. 


\section{Authors' contributions}

PG designed and conducted the cell and animal experiments, compiled and interpreted data, and wrote the paper. AJD and NC aided PG in animal treatments and tissue collection. RFF co-wrote the paper with PG. All authors were involved in revising the manuscript critically for important intellectual content, read and approved the final manuscript.

\section{Acknowledgements}

The authors would like to thank the James P. Mara Center for Lung Disease of the Pulmonary Division of St. Luke's Roosevelt Hospital for their support and Dr. Edward Eden, Dr. Gerard Turino and Dr. Charles Powell. This work was supported by grants made available to P.G. (Flight Attendant Medical Research Institute (YCSA 113380) and to R.F. (US National Institutes of Health 5R01HL098528-04)

Received: 8 May 2014 Accepted: 15 September 2014

Published online: 03 October 2014

\section{References}

1. Guo-Parke H, Canning P, Douglas I, Villenave R, Heaney LG, Coyle PV, Lyons JD, Shields MD, Power UF: Relative Respiratory Syncytial Virus Cytopathogenesis in Upper and Lower Respiratory Tract Epithelium. Am J Respir Crit Care Med 2013, 188(7):842-851.

2. Falsey AR, Hennessey PA, Formica MA, Cox C, Walsh EE: Respiratory syncytial virus infection in elderly and high-risk adults. N Engl J Med 2005, 352(17):1749-1759.

3. Respiratory syncytial virus activity-United States, 2003-2004. MMWR Morb Mortal Wkly Rep 2004, 53(49):1159-1160.

4. Hacking D, Hull J: Respiratory syncytial virus-viral biology and the host response. J Infect 2002, 45(1):18-24

5. Sigurs N, Gustafsson PM, Bjarnason R, Lundberg F, Schmidt S, Sigurbergsson F, Kjellman B: Severe respiratory syncytial virus bronchiolitis in infancy and asthma and allergy at age 13. Am J Respir Crit Care Med 2005, 171(2):137-141.

6. Sajjan US: Susceptibility to viral infections in chronic obstructive pulmonary disease: role of epithelial cells. Curr Opin Pulm Med 2013, 19(2):125-132.

7. Noah TL, Henderson FW, Wortman IA, Devlin RB, Handy J, Koren HS, Becker S: Nasal cytokine production in viral acute upper respiratory infection of childhood. J Infect Dis 1995, 171(3):584-592.

8. Sheeran P, Jafri H, Carubelli C, Saavedra J, Johnson C, Krisher K, Sanchez PJ, Ramilo O: Elevated cytokine concentrations in the nasopharyngeal and tracheal secretions of children with respiratory syncytial virus disease. Pediatr Infect Dis J 1999, 18(2):115-122.

9. Abu-Harb M, Bell F, Finn A, Rao WH, Nixon L, Shale D, Everard ML: IL-8 and neutrophil elastase levels in the respiratory tract of infants with RSV bronchiolitis. Eur Respir J 1999, 14(1):139-143.

10. Tabarani CM, Bonville CA, Suryadevara M, Branigan P, Wang D, Huang D: Rosenberg HF. Novel Inflammatory Markers, Clinical Risk Factors, and Virus Type Associated with Severe Respiratory Syncytial Virus Infection. Pediatr Infect Dis J: Domachowske JB; 2013.

11. Arnold R, Humbert B, Werchau H, Gallati H, Konig W: Interleukin-8, interleukin-6, and soluble tumour necrosis factor receptor type I release from a human pulmonary epithelial cell line (A549) exposed to respiratory syncytial virus. Immunology 1994, 82(1):126-133.

12. Zdrenghea MT, Telcian AG, Laza-Stanca $V$, Bellettato CM, Edwards MR, Nikonova A, Khaitov MR, Azimi N, Groh V, Mallia P, Johnston SL, Stanciu LA: RSV infection modulates IL-15 production and MICA levels in respiratory epithelial cells. Eur Respir J 2012, 39(3):712-720.

13. Zhang Y, Luxon BA, Casola A, Garofalo RP, Jamaluddin M, Brasier AR: Expression of respiratory syncytial virus-induced chemokine gene networks in lower airway epithelial cells revealed by cDNA microarrays. J Virol 2001, 75(19):9044-9058.

14. Dosanjh A, Rednam S, Martin M: Respiratory syncytial virus augments production of fibroblast growth factor basic in vitro: implications for a possible mechanism of prolonged wheezing after infection. Pediatr Allergy Immunol 2003, 14(6):437-440.

15. Brand HK, Ferwerda G, Preijers F, de Groot R, Neeleman C, Staal FJ, Warris A, Hermans PW: CD4+ T-cell counts and interleukin-8 and CCL-5 plasma concentrations discriminate disease severity in children with RSV infection. Pediatr Res 2013, 73(2):187-193.
16. Kim CK, Callaway Z, Koh YY, Kim SH, Fujisawa T: Airway IFN-gamma production during RSV bronchiolitis is associated with eosinophilic inflammation. Lung 2012, 190(2):183-188.

17. Ermers MJ, Janssen R, Onland-Moret NC, Hodemaekers HM, Rovers MM, Houben ML, Kimpen JL, Bont LJ: IL10 family member genes IL19 and IL20 are associated with recurrent wheeze after respiratory syncytial virus bronchiolitis. Pediatr Res 2011, 70(5):518-523.

18. Wright C, Oliver KC, Fenwick Fl, Smith NM, Toms GL: A monoclonal antibody pool for routine immunohistochemical detection of human respiratory syncytial virus antigens in formalin-fixed, paraffin-embedded tissue. J Pathol 1997, 182(2):238-244.

19. McGill JL, Nonnecke BJ, Lippolis JD, Reinhardt TA, Sacco RE: Differential chemokine and cytokine production by neonatal bovine gammadelta T-cell subsets in response to viral toll-like receptor agonists and in vivo respiratory syncytial virus infection. Immunology 2013, 139(2):227-244.

20. Kimura H, Yoshizumi M, Ishii H, Oishi K, Ryo A: Cytokine production and signaling pathways in respiratory virus infection. Front Microbiol 2013, 4:276.

21. Kang MJ, Lee CG, Lee JY, Dela Cruz CS, Chen ZJ, Enelow R, Elias JA: Cigarette smoke selectively enhances viral PAMP- and virus-induced pulmonary innate immune and remodeling responses in mice. J Clin Invest 2008, 118(8):2771-2784.

22. Slater L, Bartlett NW, Haas JJ, Zhu J, Message SD, Walton RP, Sykes A, Dahdaleh S, Clarke DL, Belvisi MG, Kon OM, Fujita T, Jeffery PK, Johnston SL, Edwards MR: Co-ordinated role of TLR3, RIG-I and MDA5 in the innate response to rhinovirus in bronchial epithelium. PLOS Pathog 2010, 6(11):e1001178.

23. Scagnolari C, Midulla F, Pierangeli A, Moretti C, Bonci E, Berardi R, De Angelis D, Selvaggi C, Di Marco P, Girardi E, Antonelli G: Gene expression of nucleic acid-sensing pattern recognition receptors in children hospitalized for respiratory syncytial virus-associated acute bronchiolitis. Clin Vaccine Immunol 2009, 16(6):816-823.

24. Kurt-Jones EA, Popova L, Kwinn L, Haynes LM, Jones LP, Tripp RA, Walsh EE, Freeman MW, Golenbock DT, Anderson L, Finberg RW: Pattern recognition receptors TLR4 and CD14 mediate response to respiratory syncytial virus. Nat Immunol 2000, 1(5):398-401.

25. Sasai M, Shingai M, Funami K, Yoneyama M, Fujita T, Matsumoto M, Seya T: NAK-associated protein 1 participates in both the TLR3 and the cytoplasmic pathways in type I IFN induction. J Immunol 2006, 177(12):8676-8683.

26. Shafique M, Meijerhof T, Wilschut J, de Haan A: Evaluation of an intranasal virosomal vaccine against respiratory syncytial virus in mice: effect of TLR2 and NOD2 ligands on induction of systemic and mucosal immune responses. PLoS One 2013, 8(4):e61287.

27. Williams RL, Hilton DJ, Pease $S$, Willson TA, Stewart $C L$, Gearing DP, Wagner EF, Metcalf D, Nicola NA, Gough NM: Myeloid leukaemia inhibitory factor maintains the developmental potential of embryonic stem cells. Nature 1988, 336(6200):684-687.

28. Jorens PG, De Jongh $R$, Bossaert LL, De Backer W, Herman AG, Pollet $H$, Bosmans E, Taupin JL, Moreau JF: High levels of leukaemia inhibitory factor in ARDS. Cytokine 1996, 8(11):873-876.

29. Quinton $\sqcup$, Mizgerd JP, Hilliard KL, Jones MR, Kwon CY, Allen E: Leukemia inhibitory factor signaling is required for lung protection during pneumonia. J Immuno/ 2012, 188(12):6300-6308.

30. Loo YM, Fornek J, Crochet N, Bajwa G, Perwitasari O, Martinez-Sobrido L, Akira S, Gill MA, Garcia-Sastre A, Katze MG, Gale M Jr: Distinct RIG-I and MDA5 signaling by RNA viruses in innate immunity. J Virol 2008, 82(1):335-345.

31. Lay MK, Gonzalez PA, Leon MA, Cespedes PF, Bueno SM, Riedel CA, Kalergis AM: Advances in understanding respiratory syncytial virus infection in airway epithelial cells and consequential effects on the immune response. Microbes Infect 2013, 15(3):230-242.

32. Ulich TR, Fann MJ, Patterson PH, Williams JH, Samal B, Del Castillo J, Yin S, Guo K, Remick DG: Intratracheal injection of LPS and cytokines. V LPS induces expression of LIF and LIF inhibits acute inflammation Am J Physiol 1994, 267(4 Pt 1):L442-L446.

33. Wang J, Chen Q, Corne J, Zhu Z, Lee CG, Bhandari V, Homer RJ, Elias JA: Pulmonary expression of leukemia inhibitory factor induces $B$ cell hyperplasia and confers protection in hyperoxia. J Biol Chem 2003, 278(33):31226-31232. 
34. Tripp RA, Oshansky C, Alvarez R: Cytokines and respiratory syncytial virus infection. Proc Am Thorac Soc 2005, 2(2):147-149.

35. Jafri HS, Chavez-Bueno S, Mejias A, Gomez AM, Rios AM, Nassi SS, Yusuf M, Kapur P, Hardy RD, Hatfield J, Rogers BB, Krisher K, Ramilo O: Respiratory syncytial virus induces pneumonia, cytokine response, airway obstruction, and chronic inflammatory infiltrates associated with long-term airway hyperresponsiveness in mice. J Infect Dis 2004, 189(10):1856-1865.

36. McNamara PS, Flanagan BF, Hart CA, Smyth RL: Production of chemokines in the lungs of infants with severe respiratory syncytial virus bronchiolitis. J Infect Dis 2005, 191(8):1225-1232.

37. Bach JP, Rinn B, Meyer B, Dodel R, Bacher M: Role of MIF in inflammation and tumorigenesis. Oncology 2008, 75(3-4):127-133.

38. Chen L, Lin SX, Agha-Majzoub R, Overbergh L, Mathieu C, Chan LS: CCL27 is a critical factor for the development of atopic dermatitis in the keratin-14 IL-4 transgenic mouse model. Int Immunol 2006, 18(8):1233-1242.

39. Qiu L, Huang D, Chen CY, Wang R, Shen L, Shen Y, Hunt R, Estep J, Haynes BF, Jacobs WR Jr, Letvin N, Du G, Chen ZW: Severe tuberculosis induces unbalanced up-regulation of gene networks and overexpression of IL-22, MIP-1alpha, CCL27, IP-10, CCR4, CCR5, CXCR3, PD1, PDL2, IL-3, IFN-beta, TIM1, and TLR2 but low antigen-specific cellular responses. I Infect Dis 2008, 198(10):1514-1519.

40. Elliott MB, Tebbey PW, Pryharski KS, Scheuer CA, Laughlin TS, Hancock GE: Inhibition of respiratory syncytial virus infection with the CC chemokine RANTES (CCL5). J Med Virol 2004, 73(2):300-308.

41. Bleul CC, Fuhlbrigge RC, Casasnovas JM, Aiuti A, Springer TA: A highly efficacious lymphocyte chemoattractant, stromal cell-derived factor 1 (SDF-1). J Exp Med 1996, 184(3):1101-1109.

42. Makino H, Aono Y, Azuma M, Kishi M, Yokota Y, Kinoshita K, Takezaki A, Kishi J, Kawano H, Ogawa H, Uehara H, Izumi K, Sone S, Nishioka Y: Antifibrotic effects of CXCR4 antagonist in bleomycin-induced pulmonary fibrosis in mice. J Med Invest 2013, 60(1-2):127-137.

43. Hiraoka A, Yano Ki K, Kagami N, Takeshige K, Mio H, Anazawa H, Sugimoto S: Stem cell growth factor: in situ hybridization analysis on the gene expression, molecular characterization and in vitro proliferative activity of a recombinant preparation on primitive hematopoietic progenitor cells. Hematol J 2001, 2(5):307-315.

44. Makowska JS, Cieslak M, Kowalski ML: Stem cell factor and its soluble receptor (c-kit) in serum of asthmatic patients- correlation with disease severity. BMC Pulm Med 2009, 9:27.

45. Linker RA, Kruse N, Israel S, Wei T, Seubert S, Hombach A, Holtmann B, Luhder F, Ransohoff RM, Sendtner M, Gold R: Leukemia inhibitory factor deficiency modulates the immune response and limits autoimmune demyelination: a new role for neurotrophic cytokines in neuroinflammation. J Immunol 2008, 180(4):2204-2213.

46. Ward NS, Waxman AB, Homer RJ, Mantell LL, Einarsson O, Du Y, Elias JA: Interleukin-6-induced protection in hyperoxic acute lung injury. Am 」 Respir Cell Mol Biol 2000, 22(5):535-542.

47. Waxman $A B$, Einarsson $O$, Seres $T$, Knickelbein RG, Warshaw JB, Johnston $R$, Homer RJ, Elias JA: Targeted lung expression of interleukin-11 enhances murine tolerance of $100 \%$ oxygen and diminishes hyperoxia-induced DNA fragmentation. J Clin Invest 1998, 101(9):1970-1982.

48. Kamohara H, Sakamoto K, Ishiko T, Masuda Y, Abe T, Ogawa M: Leukemia inhibitory factor induces apoptosis and proliferation of human carcinoma cells through different oncogene pathways. Int I Cancer 1997, 72(4):687-695.

49. Schere-Levy C, Buggiano V, Quaglino A, Gattelli A, Cirio MC, Piazzon I, Vanzulli S, Kordon EC: Leukemia inhibitory factor induces apoptosis of the mammary epithelial cells and participates in mouse mammary gland involution. Exp Cell Res 2003, 282(1):35-47.

50. Furue M, Okamoto T, Hayashi Y, Okochi H, Fujimoto M, Myoishi Y, Abe T, Ohnuma K, Sato GH, Asashima M, Sato JD: Leukemia inhibitory factor as an anti-apoptotic mitogen for pluripotent mouse embryonic stem cells in a serum-free medium without feeder cells. In Vitro Cell Dev Biol Anim 2005, 41(1-2):19-28.

51. Lu Y, Fukuyama S, Yoshida R, Kobayashi T, Saeki K, Shiraishi H, Yoshimura A, Takaesu G: Loss of SOCS3 gene expression converts STAT3 function from anti-apoptotic to pro-apoptotic. J Biol Chem 2006, 281(48):36683-36690

52. Jeffery PK, Wardlaw AJ, Nelson FC, Collins JV, Kay AB: Bronchial biopsies in asthma. An ultrastructural, quantitative study and correlation with hyperreactivity. Am Rev Respir Dis 1989, 140(6):1745-1753.
53. Hogan BL, Barkauskas CE, Chapman HA, Epstein JA, Jain R, Hsia CC, Niklason L, Calle E, Le A, Randell SH, Rock J, Snitow M, Krummel M, Stripp BR, Vu T, White ES, Whitsett JA, Morrisey EE: Repair and Regeneration of the Respiratory System: Complexity, Plasticity, and Mechanisms of Lung Stem Cell Function. Cell Stem Cell 2014, 15(2):123-138.

54. Tadokoro $T$, Wang $Y$, Barak LS, Bai $Y$, Randell SH, Hogan BL: IL-6/ STAT3 promotes regeneration of airway ciliated cells from basal stem cells. Proc Natl Acad Sci U S A 2014, 111(35):E3641-E3649.

55. Manuse MJ, Parks GD: TLR3-dependent upregulation of RIG-I leads to enhanced cytokine production from cells infected with the parainfluenza virus SV5. Virology 2010, 397(1):231-241.

56. Lukacs NW, Smit JJ, Mukherjee S, Morris SB, Nunez G, Lindell DM: Respiratory virus-induced TLR7 activation controls IL-17-associated increased mucus via IL-23 regulation. J Immunol 2010, 185(4):2231-2239.

57. Rudd BD, Burstein E, Duckett CS, Li X, Lukacs NW: Differential role for TLR3 in respiratory syncytial virus-induced chemokine expression. J Virol 2005, 79(6):3350-3357.

58. Bhoj VG, Sun Q, Bhoj EJ, Somers C, Chen X, Torres JP, Mejias A, Gomez AM, Jafri H, Ramilo O, Chen ZJ: MAVS and MyD88 are essential for innate immunity but not cytotoxic T lymphocyte response against respiratory syncytial virus. Proc Natl Acad Sci U S A 2008, 105(37):14046-14051.

59. Sun Q, Sun L, Liu HH, Chen X, Seth RB, Forman J, Chen ZJ: The specific and essential role of MAVS in antiviral innate immune responses. Immunity 2006, 24(5):633-642.

60. Martinez-Gil L, Goff PH, Hai R, Garcia-Sastre A, Shaw ML, Palese P: A Sendai virus-derived RNA agonist of RIG-I as a virus vaccine adjuvant. J Virol 2013, 87(3):1290-1300.

61. Rehwinkel J, Tan CP, Goubau D, Schulz O, Pichlmair A, Bier K, Robb N, Vreede F, Barclay W, Fodor E, Reis e Sousa C: RIG-I detects viral genomic RNA during negative-strand RNA virus infection. Cell 2010, 140(3):397-408.

62. Schlee M, Roth A, Hornung V, Hagmann CA, Wimmenauer V, Barchet W, Coch C, Janke M, Mihailovic A, Wardle G, Juranek S, Kato H, Kawai T, Poeck H, Fitzgerald KA, Takeuchi O, Akira S, Tuschl T, Latz E, Ludwig J, Hartmann G: Recognition of $5^{\prime}$ triphosphate by RIG-I helicase requires short blunt double-stranded RNA as contained in panhandle of negative-strand virus. Immunity 2009, 31(1):25-34.

63. Boyapalle S, Wong T, Garay J, Teng M, San Juan-Vergara H, Mohapatra S: Respiratory syncytial virus NS1 protein colocalizes with mitochondrial antiviral signaling protein MAVS following infection. PLOS One 2012, 7(2):e29386.

64. Ling Z, Tran $K C$, Teng MN: Human respiratory syncytial virus nonstructural protein NS2 antagonizes the activation of beta interferon transcription by interacting with RIG-I. J Virol 2009, 83(8):3734-3742.

65. Liu P, Jamaluddin M, Li K, Garofalo RP, Casola A, Brasier AR: Retinoic acidinducible gene I mediates early antiviral response and Toll-like receptor 3 expression in respiratory syncytial virus-infected airway epithelial cells. J Virol 2007, 81(3):1401-1411.

66. Villenave R, Thavagnanam S, Sarlang S, Parker J, Douglas I, Skibinski G, Heaney LG, McKaigue JP, Coyle PV, Shields MD, Power UF: In vitro modeling of respiratory syncytial virus infection of pediatric bronchial epithelium, the primary target of infection in vivo. Proc Natl Acad Sci U S A 2012, 109(13):5040-5045

67. Zhang L, Peeples ME, Boucher RC, Collins PL, Pickles RJ: Respiratory syncytial virus infection of human airway epithelial cells is polarized, specific to ciliated cells, and without obvious cytopathology. J Virol 2002, 76(11):5654-5666.

68. Boukhvalova MS, Yim KC, Prince GA, Blanco JC: Methods for monitoring dynamics of pulmonary RSV replication by viral culture and by real-time reverse transcription-PCR in vivo: Detection of abortive viral replication. Curr Protoc Cell Biol 2010, Chapter 26:26. Unit26.

69. Foronjy RF, Taggart CC, Dabo AJ, Weldon S, Cummins N, Geraghty P: Type-I interferons induce lung protease responses following respiratory syncytial virus infection via RIG-I-like receptors. Mucosal Immunol 2014 [Epud ahead of print].

\section{doi:10.1186/s12865-014-0041-4}

Cite this article as: Foronjy et al.: Leukemia inhibitory factor protects the lung during respiratory syncytial viral infection. BMC Immunology 2014 15:41. 